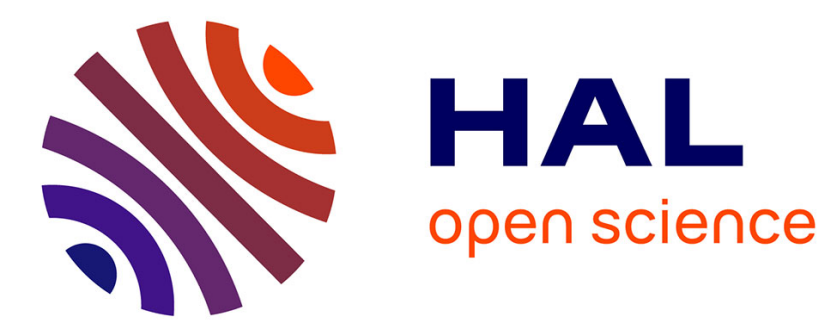

\title{
Logical modeling of emotions for Ambient Intelligence
}

Carole Adam, Benoit Gaudou, Dominique Longin, Emiliano Lorini

\section{To cite this version:}

Carole Adam, Benoit Gaudou, Dominique Longin, Emiliano Lorini. Logical modeling of emotions for Ambient Intelligence. Fulvio Mastrogiovanni; Nak-Young Chong. Handbook of Research on Ambient Intelligence: Trends and Perspectives, IGI Global Publisher, 2011, 978-1616928575. 10.4018/978-161692-857-5.ch007 . hal-00950876

\section{HAL Id: hal-00950876 https://hal.science/hal-00950876}

Submitted on 3 Dec 2021

HAL is a multi-disciplinary open access archive for the deposit and dissemination of scientific research documents, whether they are published or not. The documents may come from teaching and research institutions in France or abroad, or from public or private research centers.
L'archive ouverte pluridisciplinaire HAL, est destinée au dépôt et à la diffusion de documents scientifiques de niveau recherche, publiés ou non, émanant des établissements d'enseignement et de recherche français ou étrangers, des laboratoires publics ou privés. 


\section{Logical modeling of emotions for Ambient}

\section{Intelligence}

Carole Adam ${ }^{1}$, Benoit Gaudou ${ }^{2}$, Dominique Login ${ }^{3}$, Emiliano Lorini ${ }^{3}$

${ }^{1}$ RMIT University, Melbourne, Australia - Email: carole.adam.rmit@gmail.com

${ }^{2}$ UMI 209 UMMISCO, IRD, IFI, Hanoi, Vietnam - Email: benoit.gaudou@alumni.enseeiht.fr

${ }^{3}$ University of Toulouse, CNRS, IRIT, Toulouse, France - Emails: longin@irit.fr, $\underline{\text { lorini@irit.fr }}$

Abstract.

Ambient Intelligence (AmI) is the art of designing intelligent and user-focused environments. It is thus of great importance to take human factors into account. In this chapter we especially focus on emotions, that have been proved to be essential in human reasoning and interaction. To this end, we assume that we can take advantage of the results obtained in Artificial Intelligence about the formal modeling of emotions. This chapter specifically aims at showing the interest of logic as a tool to design agents endowed with emotional abilities useful for Ambient Intelligence applications. In particular, we show that modal logics allow the representation of the mental attitudes involved in emotions such as beliefs, goals or ideals. Moreover, we illustrate how modal logics can be used to represent complex emotions (also called self-conscious emotions) involving elaborated forms of reasoning, such as self-attribution of responsibility and counterfactual reasoning. Examples of complex emotions are regret and guilt. We illustrate our logical approach by formalizing some case studies concerning an intelligent house taking care of its inhabitants.

Keywords: emotions, intelligent agents, responsibility, modal logic. 


\section{Introduction}

Ambient Intelligence (AmI) is the art of designing intelligent and user-focused environments, i.e. environments that can adapt their behavior to users and to their specific goals or needs at every moment, in order to insure their well-being in a non-intrusive and nearly invisible way. AmI systems should be embedded, context aware, personalized and adaptive [34]. AmI is a highly multidisciplinary field of research, at the convergence between as various disciplines as for instance electronics, networks, ergonomics, robotics or computer science. Some examples of applications are ISTAG's research scenarios [21], industrial ones such as Philips intelligent house HomeLab [1], or academic projects such as MIT's Oxygen project and their MediaLab.

Since the focus of AmI is on the human users, it is of great importance in order to better understand them to have a model of human factors and to take these factors into account [60]. In this chapter we focus on a particular human factor, namely emotions, that have been proven to be essential in the human decision making process [18], but also in the interaction between users and machines [48]. Therefore we think that AmI can take advantage of the results obtained in Affective Computing [48] or Artificial Intelligence (AI) about emotions

In particular, the aim of this chapter is to show how logical tools and methods, traditionally used in the field of $\mathrm{AI}$ for the formal design of intelligent systems and agents, can be exploited in order to specify emotional agents for AmI applications. The term emotional agents refers to intelligent agents endowed with some emotional abilities, e.g. identifying and reacting to the users' emotions. These agents may also be able to "feel" emotions ${ }^{1}$, to express them, and to behave accordingly. We think that there are two main reasons justifying the use of logical methods for the design of such emotional agents.

First, although physiological sensors can detect some simple users' emotions such as happiness [50], they are neither able to determine the object of these emotions (e.g. happiness about the sun shining or about

${ }^{1}$ According to Picard: "The computer's emotions are labels for states that may not exactly match the analogous human feelings, but that initiate behavior we would expect someone in that state to display." [48, p. 298] 
being in holidays), nor able to differentiate between some close emotions such as sadness, regret and guilt [44]. Indeed, to do this, it is necessary to take into account the context and the users' mental attitudes such as beliefs, desires, ideals which are the cognitive constituents of emotions. Now, so-called BDI (Belief, Desire, Intention) logics developed in the field of AI in the last fifteen years (see e.g. [16],[52],[39]) offer expressive frameworks to represent agents' mental attitudes and to reconstruct on their basis the cognitive layer of emotions [2],[14],[57].

Second, in order to adapt to the users, an AmI system must be able to reason about their emotions and to analyze them. In particular, an AmI system must have a model of users' emotions and of the ways in which they affect their behavior, like action tendencies [28] (e.g. feeling fear entails escape) and coping strategies [37] (e.g. denying an information causing too much sadness). This model would allow the AmI system to reason about (e.g. anticipate) the effects of its own actions on the users' emotions and actions. Now, logical approaches were specifically designed to endow agents with this kind of reasoning capabilities.

The previous two arguments are even more obvious when looking at the cognitive structure of complex emotions $^{2}$ such as regret, shame, guilt, reproach because they involve complex forms of reasoning. Following [23],[35], we consider that complex emotions are those that are based on a larger set of appraisal variables $^{3}$ than the set involved in basic emotions (e.g. fear, happiness, sadness). Besides, while basic emotions are related to an individual's basic biological needs and can be elicited after a merely automatic and unconscious analysis of the situation, complex emotions are based on a conscious cognitive activity and involve very sophisticated forms of reasoning such as self-attribution of responsibility and counterfactual reasoning.

Thus finally, logical approaches seem to be a very well adapted solution for the design of AmI systems which are capable of identifying the users' complex emotions (e.g. guilt, shame), determining their object

\footnotetext{
${ }_{2}^{2}$ Some authors (e.g. [59]) prefer the term self-conscious emotion to the term complex emotion.

3 According to cognitive appraisal theories [28],[37],[46], emotions are triggered by a process (called appraisal) determining the significance of the situation for the individual by assessing it w.r.t. various criterions called appraisal variables (e.g. desirability).
} 
(e.g. why is the user feeling guilty? What is (s) he regretting for?), and reasoning about them (e.g. will (s)he feel ashamed if I tell him/her that (s)he failed his/her exam?).

The present chapter is organized as follows. In Section 2, we present a very general overview of the role of emotions in some present and future AmI applications; moreover, we discuss in more details the contribution that logical methods could bring to AmI applications. In Section 3, we present a logical framework allowing a formal specification of agents' mental attitudes and of important conceptual components of complex emotions such as the notion of responsibility; we show how this logical framework can be used to define some complex emotions like regret and guilt. Section 4 is devoted to the application of our logical framework for complex emotions to the formalization of some case studies for AmI.

\section{State of the Art}

\subsection{Applications of emotions in AmI}

Nowadays, more and more AmI systems are endowed with emotional abilities, in order to perform better in various application fields. For instance an emotional agent for AmI can:

- express emotions to reduce users' frustration when interacting with a machine, as proven by Picard [48] (e.g. Greta [19], Breazeal's Kismet [10], or Philips' i-Cat [62]);

- favor health and well-being by developing social relationships (proven by Berkman [9] to have a positive influence on health) with sick or elderly people (e.g. Wada and Shibata's Paro robot [63]);

- favor performance by decreasing stress [12], or by interacting realistically with the user in a virtual world to train him to make decisions under stress (e.g. Gratch and Marsella's Mission Rehearsal Exercise [42] for military officers, or El Jed et al. virtual world for firemen [24]);

- assist decision making in business [41] or in health care [7];

- express believable emotions to be more attractive and engaging when employed as a virtual tutor (in an educational application) [53]. 
These applications involve different kinds of emotional abilities, such as expressing emotions or being sensitive to the user's ones. In the next section we detail what we assume to be the complete list of emotional abilities that may be useful for AmI applications.

\subsection{Useful emotional abilities for AmI systems}

In this chapter, we consider AmI systems according to the multi-agents paradigm. We thus call agents the components of AmI systems. We consider an agent from a very general point of view, i.e. as an autonomous entity with some capabilities of perception of its environment, of action on it (including interaction with other agents) and of reasoning. In the following we focus on a particular type of agents, called cognitive agents (as opposed to reactive agents), whose behavior is guided by motivations and goals. One can consider an AmI system as constituted by both cognitive and reactive agents; for example a cognitive agent can lead a network of reactive sensing agents to get information about the world in order to make adapted decisions. We assume that this representation can fit every AmI system.

\subsubsection{Identifying the users' emotion}

In order to interact with the users, adapt to them or improve their well-being, an agent has to identify their emotional state. According to Searle [55], we consider emotions as intentional mental states, like belief or intention, i.e. mental states "directed at or about or of objects or states of affairs in the world" [55, p.1] Thus emotions always have an object; similar affective states but directed at no particular object will be called moods. Therefore the identification of the users' emotions comprises two steps: identifying the type of emotion (joy, sadness, anger, etc.) and identifying its object (e.g. joy about the sunny weather).

On the one hand a great amount of work already allows systems to identify the user's type of emotion from physiological data [49],[51], gestures [64], facial expressions [58], language and voice intonation [56], etc. The challenge for AmI is to perceive this physiological information in a non intrusive way, which will be made possible thanks to technological progress (e.g. seamless sensors integrated in clothes) 
On the other hand identifying the object of the user's emotion is much more difficult, since it is not physiologically measurable (joy of winning the basket-ball championship final and joy of having an accepted paper in a great conference have the same physiological manifestations); it is thus necessary to reason about the context and the users' mental attitudes to identify this object. Moreover this additional information allows the agent to identify more precisely the users' emotion [27], for example it could allow to distinguish the sadness of having lost the final from the regret for being responsible for this defeat.

\subsubsection{Computing the agent's emotion}

As shown above, various AmI applications use agents able to express emotions, and sometimes able to behave according to their emotions. An agent's emotion should be computed from perceived external stimuli, from the agent's previous emotions, and from its representation of the world as it is or as it should be (e.g. in terms of mental attitudes). Moreover, the computation of this emotion should be informed by psychological models of human emotions [37],[46] to ensure its correctness; formalizations of such models exist to this aim [2],[57]. The computation of an agent's emotion is also deeply linked with the agent's hardware capacities.

For example, the embodied conversational agent Greta [19] uses Ortony's model [45] representing emotions with two dimensions: valence (positive or negative) and time (past, present, future, indicating when the triggering stimulus occurs). A belief network is used to match a situation with an emotion; then the corresponding dimensions are computed.

In the area of pedagogical tutoring, Steve [25] is an animated agent who inhabits a 3D environment and teaches students to operate a high pressure air compressor. It is aware of the students' actions and of their effect on the environment. Its emotions, based on Ortony et al.'s theory [46], are a function of its goals and principles, but also of the students' presumed appraisal of the situation and of Steve's relationship with them. 
Once the agent is aware of its own emotions or of the users' ones, it must be able to reason about them and to behave accordingly. This is the point of the next paragraphs.

\subsubsection{Taking the user's emotion into account in reasoning and planning}

An agent for AmI should be able to take the users' emotion into account in its reasoning process in order to influence its beliefs, goals and future actions. For example an AmI agent aiming at the users' well-being should behave differently depending on the users' emotion, or at least on its valence (positive or negative) Moreover the strategies that it can use to get the users' back to a positive emotion depend on the particular negative emotion that they feel (sadness, anger, etc.). We provide examples of various strategies in Section 4.

However only few convincing works are able to accurately identify the users' emotions in a non-intrusive way, and are thus usable in an actual interaction situation. The scenarios of the e-sense project [44] give an insight into how the users' emotions could be used by AmI systems in future applications: e.g. identify and reproduce faithfully the users' emotion on their avatar to improve their immersion in a virtual world or a video game; or monitoring psychotic or paranoiac patients to detect negative emotions (in particular intense fear) and call a doctor in case of need. In this last scenario, the users' emotion drives the behavior of the AmI system by giving it new goals to achieve or actions to perform.

The agent can also use its knowledge about the users' emotion to try to help them to cope with it (in the sense of Lazarus' coping strategies [37]), i.e. to restore their well-being in stressful situations. For example in [3] we have described some case studies involving an AmI agent endowed with a logical formalization of eight basic emotions.

2.2.4. Taking the agent's emotion into account in reasoning and planning

Similarly, the agent's own emotion should also impact its reasoning and actions. First the agent should express its emotion to the users, since it is beneficial for the interaction with them [19],[48]. This emotion 
can be expressed in various ways: facial expressions, color code, gestures, language, etc. Second its emotion can influence its reasoning process, in particular by changing its beliefs or goals (for instance it can adopt the goal to interact with users when it "feels" happy). This influence can follow from the simulation of two human psychological processes: action tendencies [28] are associated to emotions to allow individuals to make quick decisions, using their emotions as a kind of heuristic (for instance fear of a spider makes one flee what is interpreted as a danger); coping strategies [37] can be planfully used to manage stressful situations (for instance one can persuade himself that the frightening spider is finally not big enough to be really dangerous, in order not to be afraid anymore).

Lots of works have been conducted in the particular area of the expression of emotions and of the development of expressive heads and robots, in particular in the Embodied Conversational Agents (ECA) community. For example, Greta [19] was used to dialogue with users in medical applications where empathy with them is crucial. Breazeal's Kismet [10] is assumed to be able to make friend with humans and influence them. Similarly, Philips has developed a robot called iCat with an articulated face able in particular to express emotions [62].

Besides, some works exist in Artificial Intelligence in order to endow agents with the ability to use coping strategies to manage their own emotions (e.g. Gratch and Marsella's EMA agent [29], or the logical formalization of coping strategies proposed in [4]). However, as far as we know, these works have not been applied to AmI yet.

\subsection{Contribution of logic to the modelling of these emotional abilities}

While several emotional abilities are subject to a great amount of work (e.g. users' emotion recognition, agent's emotional expression), some other ones have nearly not been explored yet. We assume that the tools that are currently used in AmI are not sufficient to explore some points like reasoning about emotions and identifying the user's emotions. We would thus like to show how formal logic can be an adequate tool to manage such unsolved problems. 
In particular, determining the object of the emotion is an essential but difficult problem, as pointed by [66]. We argue that endowing an AmI agent with mental attitudes, with a logical model of emotions like the one presented in the sequel of this chapter, and with the subsequent ability of logical reasoning and deduction, could be an interesting approach in addition to classical physiological sensing. Indeed when a sensor detects the physiological clues of sadness in the users, it cannot determine alone the object of this sadness. Now if the AmI agent knows the users' beliefs and preferences (e.g. users hate rain), is aware of the context (e.g. it is raining), and has a model of emotions (e.g. sadness comes from infringed preferences), it may be able to determine what has triggered the users' sadness (e.g. rain).

Besides the user's emotion may not be exactly sadness but a more complex emotion resembling sadness, like regret or remorse. Thus endowing an AmI agent with a fine-grained model of emotions, along with a knowledge base containing extensive information about norms, responsibilities, actions, context, users' profiles, etc. will allow it to finely determine the users' emotions, and thus to finely adapt to the users' needs. Thus we assume that to precisely and finely recognize both the type and object of the users' emotion, an AmI agent should jointly use physiological sensors (or any other emotional recognition system) and a model of emotions coupled with a representation of their mental attitudes and reasoning.

We can also notice that in practice, very few emotions are used in AmI. This appears as a consequence of the previous point: complex emotions like regret, remorse, shame or pride involve high level reasoning that can hardly be managed without using specific tools (like logic, as it will be shown in the next section). One can object that a few basic emotions are sufficient for the purpose of AmI. But we answer that these complex emotions are felt everyday by the human users in whose life AmI systems aim at being integrated, so they cannot just be ignored by these systems.

Finally it appears that reasoning about emotions or behavioral adaptation from emotions is subject to very little and often preliminary works. 


\subsection{Conclusion}

In this section, we have highlighted that emotions begin to be successfully used in AmI systems. Nevertheless much more can be done to integrate this essential human factor in these systems. We have thus argued that using BDI agents in AmI systems, and endowing them with a fine-grained model of emotions, expressed in a non-ambiguous logical language, can have a lot of benefits. In particular, it would allow these agents to identify more precisely the users' emotional state. In the following section we present our logical framework dedicated to the representation of emotions, and specifically of complex ones.

\section{Logical modeling of emotions}

Recently, some researchers working in the field of multi-agent systems (MAS) have been interested in developing logical frameworks for the formal specification of emotions (see [5],[38],[57] for instance). Their main concern is to exploit logical methods in order to provide a rigorous specification of how emotions should be implemented in an artificial agent. The design of agent-based systems where agents are capable to reason about and to display some kind of emotions can indeed benefit from the accuracy of logical methods. These logical approaches also aim at disambiguating the different dimensions of emotions identified in the existing psychological models of emotions (e.g. [37],[46],[54]).

Most of proposed logical frameworks for the specification of emotions are based on the so-called BDI (Belief, Desire, Intention) logics which have been developed in the last fifteen years in the MAS domain (see [16],[39],[52] for instance). BDI logics are multimodal logics in which agents' attitudes such as beliefs, goals, desires, intentions, etc. are formalized. Such concepts are generally expressed by corresponding modal operators and their interaction properties are specified.

In the following Section 3.1, we present a simple BDI language in which three kinds of agents' attitudes can be represented by corresponding modal operators: beliefs, chosen goals (or choices), and moral attitudes called ideals (or imperatives). We show how these modal operators can be combined to provide a formal characterization of some basic emotions such as sadness and joy. Then, in Section 3.2, we show how this basic BDI language can be extended with the STIT operator (operator of the logic of Seeing To It That) 
$[8],[32]$ in order to shift from basic emotions to complex emotions, and in particular to those based on selfattribution of responsibility and on counterfactual reasoning.

\subsection{A logic of agents' mental attitudes}

The basic BDI language we consider includes two kinds of operators. Operators $\mathrm{Bel}_{i}$ are used to express what a certain agent $i$ believes. Operators Choice $_{i}$ are used to denote an agent's chosen goals, that is, the goals that the agent has decided and is committed to pursue. Given an arbitrary formula $\varphi$ of the logic:

- $\quad \mathrm{Bel}_{i} \varphi$ stands for: agent $i$ believes that $\varphi$;

- Choice ${ }_{i} \varphi$ stands for: agent $i$ has the chosen goal that $\varphi$ (which can be shortened to: agent $i$ wants $\varphi$ to be true).

These two modal operators have been extensively studied in the field of formal philosophy and AI.

Operators for belief of the form Bel $_{i}$ are doxastic operators in Hintikka's style ${ }^{4}$ [30] with a standard KD45 axiomatization [15]. This means that every operator $\mathrm{Bel}_{i}$ is supposed to be normal ${ }^{5}$, and that an agent cannot have contradictory beliefs:

$$
\neg\left(\operatorname{Bel}_{i} \varphi \wedge \mathrm{Bel}_{i} \neg \varphi\right)
$$

Moreover, beliefs satisfy the properties of positive and negative introspection:

$$
\begin{aligned}
& \mathrm{Bel}_{i} \varphi \rightarrow \mathrm{Bel}_{i} \mathrm{Bel}_{i} \varphi \\
& \neg \mathrm{Bel}_{i} \varphi \rightarrow \mathrm{Bel}_{i} \neg \mathrm{Bel}_{i} \varphi
\end{aligned}
$$

Operators for chosen goal of the form Choice $_{i}$ are similar to the operators introduced in [16]. These operators are commonly defined as $\mathrm{KD}$ operators [15], that is, every operator Choice ${ }_{i}$ is normal ${ }^{6}$, and an

\footnotetext{
${ }^{4}$ We highlight the fact that in this formalism an agent belief is something that this agent is sure about: beliefs are the subjective representation of the world for the agent. This sense is quite different from the common use of this concept: to believe a proposition $p$ often means that $p$ is uncertain.

${ }^{5}$ That is, every operator $\mathrm{Bel}_{i}$ satisfies the so-called Axiom $\mathrm{K}\left(\operatorname{Bel}_{i} \varphi \wedge \operatorname{Bel}_{i}(\varphi \rightarrow \psi) \rightarrow \operatorname{Bel}_{i} \psi\right)$ and the so-called rule of necessitation: if $\varphi$ is a theorem then $\operatorname{Bel}_{i} \varphi$ is a theorem.

${ }^{6}$ Every operator Choice ${ }_{i}$ satisfies Axiom K $\left(\right.$ Choice $_{i} \varphi \wedge$ Choice $_{i}(\varphi \rightarrow \psi) \rightarrow$ Choice $\left._{i} \psi\right)$ and the rule of necessitation: if $\varphi$ is a theorem then Choice $_{i} \varphi$ is a theorem.
} 
agent cannot have contradictory chosen goals:

$\neg\left(\right.$ Choice $_{i} \varphi \wedge$ Choice $\left._{i} \neg \varphi\right)$

DChoice

A general hypothesis about the relationship between chosen goals and beliefs is the so-called assumption of weak realism [43]. According to this hypothesis, an agent cannot choose $\varphi$ if it believes that $\varphi$ is an impossible state of affairs. This hypothesis is expressed by the following logical axiom:

$$
\text { Choice }_{i} \varphi \rightarrow \neg \text { Bel }_{i} \neg \varphi
$$

\section{WeakRealism}

Principles DChoice and WeakRealism are standard principles of rationality for chosen goals. As we said above, chosen goals (represented by operators Choice ${ }_{i}$ ) refer for us to states of affairs that the agent has decided and is committed to pursue: chosen goals are the product of an agent's rational deliberation. This is the reason why they have to be consistent (i.e. a rational agent cannot decide to pursue inconsistent states of affairs) and they have to be compatible with the agent's beliefs (i.e. a rational agent cannot decide to pursue something that it believes to be impossible).

We add another type of operator to our BDI language to talk about an agent's moral attitudes, after supposing that agents are capable to discern what (from their point of view) is morally right from what is morally wrong. This is a necessary step towards an analysis of complex emotions such as guilt and shame which involve a moral dimension. Our operators for agents' moral attitudes are of the form Ideal $_{i}$ where Ideal $_{i} \varphi$ means: $\varphi$ is an ideal state of affairs for agent $i$. More generally, Ideal $_{i} \varphi$ expresses that agent $i$ thinks that it ought to promote the realization of $\varphi$, that is, agent $i$ conceives a demanding connection between itself and the state of affairs $\varphi$. When agent $i$ endorses the ideal that $\varphi$ (i.e. Ideal $_{i} \varphi$ is true), it means that $i$ addresses a command to itself, or a request or an imperative to achieve $\varphi$ (when $\varphi$ is actually false) or to maintain $\varphi$ (when $\varphi$ is actually true) [13]. In this sense, $i$ feels morally responsible for the realization of $\varphi$.

There are different ways to explain how a state of affairs $\varphi$ becomes an ideal state of affairs of an agent. A plausible explanation is based on the hypothesis that ideals are just social norms internalized (or adopted) 
by an agent [17]. Suppose that an agent believes that in a certain group (or institution) there exists a certain norm (e.g. an obligation) prescribing that a state of affairs $\varphi$ should be true. Moreover, assume that the agent identifies itself as a member of this group. In this case, the agent adopts the norm, that is, the external norm becomes an ideal of the agent. For example, since I believe that in Italy it is obligatory to pay taxes and I identify myself as an Italian citizen, I adopt this obligation by imposing the imperative to pay taxes to myself.

In some particular cases, if an agent $i$ endorses the ideal that $\varphi$, then agent $i$ is committed to promote the realization of $\varphi$ by acting either in order to achieve $\varphi$ (when $\varphi$ is currently false) or in order to maintain $\varphi$ (when $\varphi$ is actually true). Note that this property does not reflect the general case, that is, it is not valid for all agents but only for a particular type of agents. This is the reason why we do not suppose it as a logical axiom.

However, not all chosen goals of an agent have a normative origin. A part of an agent's chosen goals are not ideals of the agent, but originate from the agent's desires. In agreement with the Humean conception [33], we conceive a desire as an agent's attitude which consists in an anticipatory mental representation of a pleasant state of affairs $\varphi$ (representational dimension of desires) that motivates the agent to achieve $\varphi$ (motivational dimension of desires). In this perspective, the motivational dimension of an agent's desire is realized through its representational dimension. For example when I desire to be at the Japanese restaurant eating sushi, I imagine myself eating sushi at the Japanese restaurant and this representation gives me pleasure. This pleasant representation motivates me to go to the Japanese restaurant.

It is out of the scope of this chapter to introduce modal operators for characterizing agents' desires and to build a model which explains how an agent's chosen goals originate from its ideals and desires. However, note that some filter of deliberation is needed to obtain a final consistent set of chosen goals which are compatible with the agent's beliefs.

The three operators for beliefs, chosen goals and ideals introduced above can be used to formalize some basic emotions such as joy and sadness.

The fact that an agent $i$ feels joy about a fact $\varphi$ (or rejoices about $\varphi$ ) can be expressed as follows: 
$\mathrm{Joy}_{i} \varphi=_{\text {def }} \operatorname{Bel}_{i} \varphi \wedge$ Choice $_{i} \varphi$

According to this definition, agent $i$ feels joy about $\varphi$ if and only if $i$ believes that $\varphi$ is true and wants $\varphi$ to be true. For example, agent $i$ feels joy for having passed the exam because $i$ believes that he has passed the exam and wants to pass the exam. In this sense, $i$ is pleased by the fact that it believes to have achieved what it was committed to achieve. In this sense, joy has a positive valence, that is, it is associated with goal achievement. $^{7}$

The fact that an agent $i$ feels sadness about a fact $\varphi$ can be expressed as follows:

$$
\operatorname{Sadness}_{i} \varphi=_{\text {def }} \operatorname{Bel}_{i} \varphi \wedge \text { Choice }_{i} \neg \varphi
$$

According to this definition, agent $i$ feels sadness about $\varphi$ if and only if $i$ believes that $\varphi$ is true and wants $\neg \varphi$ to be true. Agent $i$ feels sad for not having passed the exam because $i$ believes that he has not passed the exam and wants to pass the exam. In this sense, $i$ is displeased by the fact that it believes not to have achieved what it was committed to achieve. Thus, sadness has a negative valence, that is, it is associated with goal frustration.

In the following section we extend our basic language of beliefs, choices and ideals ${ }^{8}$ in order to provide a logical characterization of some complex emotions, namely regret and guilt.

\subsection{A logic of complex emotions}

Although the application of logical methods to the formal specification of emotions has been quite successful [4],[38],[57], we think that there is still much work to be done in the field of computational and logical modeling of complex emotions such as regret, shame, guilt or reproach. By 'complex emotions' we mean those emotions that are based on complex forms of reasoning and on a larger set of appraisal variables

\footnotetext{
${ }^{7}$ The terms positive valence and negative valence are used by Ortony et al. [46], whereas Lazarus [37] uses the terms goal congruent vs. goal incongruent emotions.

${ }^{8}$ Note that this simple language could be extended by a concept of graded belief in order to account for the intensity of emotions, that could then depend on the degree of certainty of the beliefs involved in their definition.
} 
than the set involved in 'basic emotions' (e.g. fear, happiness, sadness). According to some psychological theories of emotions (see e.g. [22],[35]), while basic emotions are related to an individual's basic biological needs and can be elicited after merely an automatic and unconscious analysis of a situation, complex emotions are based on conscious cognitive activity and involve very sophisticated forms of reasoning, in particular self-attribution of responsibility and counterfactual reasoning. For example, an agent regrets something it did, if it is aware to have taken a certain decision which turned out to be not as good as expected, and it believes that if it had decided to do something different, it would have achieved better (hence it feels responsible). In this sense, regret is based on the capacity to imagine alternative scenarios that could have occurred if it had behaved differently [36],[65]. Several psychologists working on guilt (see e.g. [37],[59]) have stressed that this emotion involves the conviction of being responsible for having injured someone or violated some norm or imperative.

Therefore, in order to build a logical framework which allows us to characterize complex emotions, it is necessary to go beyond the standard BDI approach presented in Section 3.1. Indeed, a logic of complex emotions should be expressive enough to characterize not only different types of agents' mental attitudes (beliefs, desires, goals, ideals), but also the concept of responsibility. We think that a promising solution for developing a logic of complex emotions is to combine a BDI logic, as the one presented in Section 3.1, with a logic of cooperation and multi-agent interaction [6],[8],[32],[40],[47]. Indeed, this kind of logics is well suited to support both counterfactual reasoning and reasoning about responsibility of single agents and of groups of agents. The following section is devoted to present a specific logic of cooperation and multi-agent interaction: STIT logic (the logic of Seeing To It That), that we think to be a natural candidate for a logical analysis of complex emotions.

\subsubsection{A general overview of STIT logic}

The modal logic of Seeing To It That (STIT) has been proposed in the domain of formal philosophy in the 90ies [8],[31],[32]. More recently, it has been imported into the field of theoretical computer science where its formal relationships with other logics for multi-agent systems have been studied [11]. STIT is a logic 
which supports reasoning about actions of agents and joint actions of groups. Moreover, it supports reasoning about individual powers of agents and collective powers of groups, and responsibilities of agents and groups.

The semantics of STIT is based on a branching time structure with ordered moments. We here prefer to consider the syntactical aspects of STIT rather than presenting its semantics (that is presented and extensively discussed in [8],[32],[40]).

Below $A G T$ denotes the set of all agents. $C \subseteq A G T$ denotes an arbitrary set of agents. $A G T \backslash C$ denotes the complement of the set of agents $C$ with respect to $A G T$, and $A G T \backslash\{i\}$ denotes the complement of $\{i\}$ with respect to $A G T$. In the sequel, we refer to sets of agents as groups.

In STIT logic operators of the form Stit $_{\{i\}}$ for any individual agent $i$ and Stit $_{C}$ for any group of agents $C$ are introduced. The modal formulas $\operatorname{Stit}_{\{i\}} \varphi$ and $\operatorname{Stit}_{C} \varphi$ respectively express that "a certain agent $i$ brings it about that the state of affairs $\varphi$ is true no matter what the other agents do", and "a group of agents $C$ brings it about that the state of affairs $\varphi$ is true no matter what the agents outside $C$ do". Indeed, the formulas $\mathrm{Stit}_{\{i\}} \varphi$ and $\mathrm{Stit}_{C} \varphi$ can be respectively rephrased as follows:

- cagent $i$ has just performed some action $\delta_{i}$ and, for all joint actions $\delta{ }_{A G T\{i\}}$ of the group of agents $A G T \backslash\{i\}$, if $i$ does $\delta_{i}$ while the group of agents $A G T \backslash\{i\}$ does $\delta_{A G T\{i\}}$, then $\varphi$ will be true";

"the agents in the group of agents $C$ have just performed some joint action $\delta_{C}$ and, for all joint actions $\delta^{\prime}{ }_{A G T \backslash C}$ of the agents in $A G T \backslash C$, if the agents in group $C$ do the joint action $\delta_{C}$ while the agents in the group $A G T \backslash C$ do the joint action $\delta^{\prime}{ }_{A G T \backslash C}$ then $\varphi$ will be true";

The STIT expression $\neg \mathrm{Stit}_{A G T \backslash\{i\}} \varphi$ just says that:

- the complement of $\{i\}$ with respect to $A G T$ (i.e. $A G T \backslash\{i\}$ ) does not see to it that $\varphi$ (given what the agents in $A G T \backslash\{i\}$ have chosen to do)

which is the same thing as saying that:

- given what the agents in $A G T \backslash\{i\}$ have decided to do, there exists an alternative action of agent $i$ such that, if agent $i$ had chosen this action, the state of affairs $\varphi$ would not be true now. 
If $\varphi$ is true, the latter sentence just means that agent i could have prevented the state of affairs $\varphi$ to be true now. This expresses a general concept of responsibility. Intuitively, an agent $i$ is said to be responsible for a certain state of affairs $\varphi$ if and only if:

- the state of affairs $\varphi$ is true now and

- $\quad$ agent $i$ could have prevented $\varphi$ from being true now.

Formally:

$$
\operatorname{Resp}_{i} \varphi=_{\text {def }} \varphi \wedge \neg \operatorname{Stit}_{A G T \backslash\{i\}} \varphi
$$

where $\operatorname{Resp}_{\mathrm{i}} \varphi$ means that "agent $i$ is responsible for $\varphi$ ". For example, imagine that there are two agents $A G T$ $=\{$ Bill, Bob $\}$ who have to take care of a plant. Both Bill and Bob have only two actions available: water the plant or do nothing. If either both Bill and Bob water the plant or both Bill and Bob do nothing, the plant will die. In the former case the plant will die because it does not tolerate too much water. In the latter case it will die because it lacks water. If Bill (resp. Bob) waters the plant and Bob (resp. Bill) does nothing, the plant will survive. Suppose both Bill and Bob decide to water the plant and, as a consequence of their choices, the plant dies. In this situation both Bill and Bob are responsible for the death of the plant: $\operatorname{Resp}_{\text {Bill }}$ deadPlant $\wedge \operatorname{Resp}_{\text {Bob }}$ deadPlant. This means that: the plant is dead and, given what Bill had decided to do (i.e. watering the plant), there exists an alternative action of Bob (i.e. doing nothing) such that, if Bob did perform this action, the plant would have survived; and given what Bob had decided to do (i.e. watering the plant), there exists an alternative action of Bill (i.e. doing nothing) such that, if Bill did perform this action, the plant would have survived. In this sense, Bill (resp. Bob) could have avoided the death of the plant. This is the reason why both Bill and Bob are responsible for it.

\subsubsection{A formalization of regret and guilt}

As said before, an integration of STIT logic with a logic of agents' mental attitudes is particularly suitable to provide a logical characterization of complex emotions based on the concept of responsibility. Here we just consider the emotions of regret and guilt as case studies.

We add modal operators for beliefs, goals and ideals as the ones presented in Section 3.1 to STIT logic. 
Then we can come up with the following formal characterization of the concept of regret and guilt.

We say that $i$ regrets for $\varphi$ if and only if $\neg \varphi$ is a chosen goal of $i$ and $i$ believes that it is responsible for $\varphi$. Formally:

$$
\operatorname{Regret}_{i} \varphi=_{\text {def }} \operatorname{Bel}_{i} \operatorname{Resp}_{i} \varphi \wedge \text { Choice }_{i} \neg \varphi
$$

Imagine a situation in which there are only two agents $i$ and $j$, that is, $A G T=\{i, j\}$. Agent $i$ decides to park its car in a no parking area. Agent $j$ (the policeman) fines agent $i 100 €$. Agent $i$ regrets for having been fined $100 €$ (noted Regret ${ }_{i}$ fine). This means that, $i$ wants not to be fined (noted Choice $\neg \neg$ fine) and believes

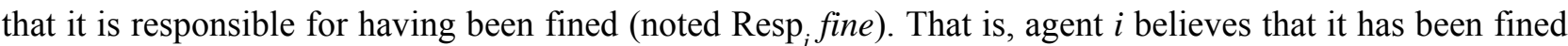
$100 €$ and believes that it could have avoided to be fined (by parking elsewhere).

It is worth noting that, according to the definition of regret given here and the definition of sadness given in Section 3.1, the former emotion entails the latter. That is, if agent $i$ regrets for $\varphi$ then, it feels sad about $\varphi$. Formally:

$$
\operatorname{Regret}_{i} \varphi \rightarrow \operatorname{Sadness}_{i} \varphi
$$

The concept of responsibility is also relevant for the characterization of the guilt emotion. Indeed, guilt involves the belief of being responsible for the violation of an ideal or imperative. Formally:

$$
\operatorname{Guilt}_{i} \varphi={ }_{\text {def }} \operatorname{Bel}_{i} \operatorname{Resp}_{i} \varphi \wedge \text { Ideal }_{i} \neg \varphi
$$

According to this definition, agent $i$ feels guilty for $\varphi$ (noted Guilt ${ }_{i} \varphi$ ) if and only if $\neg \varphi$ is an ideal state of affairs for $i$ (noted Ideal ${ }_{i} \neg \varphi$ ) and $i$ believes to be responsible for $\varphi$.

For example, imagine a situation in which there are only two agents $i$ and $j$ (that is $A G T=\{i, j\}$ ). Agent $i$ decides to shoot with gun and accidentally kills agent $j$. Agent $i$ feels guilty for having killed someone (noted Guilt ${ }_{i}$ killedSomeone). This means that, $i$ addresses an imperative to itself not to kill other people (noted Ideal $_{i} \neg$ killedSomeone) and agent $i$ believes that it is responsible for having killed someone (noted

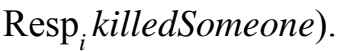


As said above, in some situations it is reasonable to suppose that an agent decides to pursue all its ideals, that is, if $\varphi$ is an ideal state of affairs for agent $i$ then, $i$ chooses $\varphi$. Under this hypothesis, we can infer formally that guilt implies regret, that is:

$$
\text { Guilt }_{i} \varphi \rightarrow \operatorname{Regret}_{i} \varphi
$$

This is not the place to defend the plausibility of such a formal consequence. We think that the only way to validate it is to perform psychological experiments on human subjects.

\subsection{Related works on logical modeling of emotions}

As emphasized in the previous sections, other researchers have exploited logical methods in order to build formal models of emotions and affective agents. One of the most prominent logical account of emotions is the one proposed in [57]. Meyer et al. provide a logical analysis of several types of emotions grounded on existing psychological theories of emotions (e.g. [37],[46]). To this aim, a logical framework which support reasoning about actions, time and agents' attitudes is used.

It is worth noting that there is a fundamental difference between our approach and Meyer et al.'s approach. In Meyer et al.'s approach each instance of emotion is represented with a special predicate, or fluent, in the jargon of reasoning about action and change, to indicate that these predicates change over time. For every fluent a set of effects of the corresponding emotions on the agent's planning strategies are specified, as well as the preconditions for triggering the emotion. On the contrary, in our logic of emotions there are no specific formal constructs which are used to denote that a certain emotion arises at a certain time. We just define emotions from more basic concepts of knowledge and desire. For instance, according to our definition of regret, an agent regrets for $\varphi$ if and only if it has the chosen goal that $\neg \varphi$ and believes that it is responsible for $\varphi$. We prefer this kind of approach since it allows to characterize explicitly the cognitive constituents of emotions.

Nevertheless, we are aware that our approach is simplistic. In particular, it misses important psychological aspects such as the fact that the cognitive constituents of emotions (e.g. knowledge, desires) are usually joined with bodily activation and components, and these components shape the whole subjective state and 
determine the nature of the affective reaction [28]. We deal here with the limits of any disembodied mind (and model).

\section{Case study}

In order to illustrate how an agent able to reason about users' complex emotions can be useful in AmI, we present here several scenarios involving such an agent in the context of an intelligent house. There already exist some industrial or academic projects aiming at designing such intelligent houses, for example SGI Japan RoomRender, Philips HomeLab [1] or MIT MediaLab. We suppose that this intelligent house is able to monitor its inhabitants (a family) in order to measure some physiological data that will be useful to identify their emotion. But if this physiological data allows to identify some emotions [27],[48],[51], information about the context and users' mental attitudes allows to be more precise [27]. The house software is thus also composed of several agents integrated in various objects (the fridge, a personal assistant, a car...). Each agent has access to a profile of the inhabitants, including their desires, beliefs, and any useful information; it is also endowed with a logical model of emotions like the one proposed in this chapter. It is then able to reason about all this information in order to precisely identify the users' emotion and its object, and to decide of its subsequent behavior.

To be more precise, an agent first detects an emotion and then tries to refine and explain it a posteriori from its knowledge base and its model of emotions; it does not predict emotions, which could lead to unadapted reactions in case of wrong prediction. As a very simple example, an agent can detect a negatively valenced emotion in the mother; then it observes that it is raining and that it is contrary to the mother's preferences; thus it infers from its model of emotions that she may be sad about the weather; and finally it performs some actions to divert her from the rain outside (for instance, display some photos of sunny landscapes on the wall, play music, etc.).

The two scenarios detailed below illustrate the two complex emotions that we have formalized in the previous section: regret and guilt. 


\subsection{Scenario 1: regret}

A morning, the father $f$ is in a hurry because of an important meeting with his boss and a big client. He thus decides to use his car instead of the subway he usually takes, in order to gain some time. But he is caught into traffic jam because of an accident that just occurred five minutes ago. He finally arrives later than with the subway, and he is five minutes late for the meeting. He then reasons about his alternative plan (take the subway) and its supposed outcome (arrive on time) that would have been better than the outcome of his actual plan (arrive late, by car). He thus feels regret about being late because he is responsible for the wrong choice that led to this undesirable situation

We now assume that the father is equipped with a kind of personal assistant disposing of all useful information about him and able to reason about his mental attitudes and emotions. This agent detects a negative emotion from $f$ 's physiological data, and then tries to refine and explain it from its knowledge base and its logical model of emotions. In particular in this context this agent knows ${ }^{9}$ that:

- $\quad$ the father $f$ aimed to arrive on time (before 9 a.m.) (Choice $f$ isLate);

- $f$ failed to achieve his goal (he arrived late, at 9.05 a.m.) (isLate) and is aware of his failure

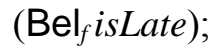

- $\quad f$ believes that he could have seen to it not to be late (Bel $\mathrm{B}_{f} \mathrm{Stit}_{A G T \backslash\{f\}}$ isLate): he could have taken the subway which would have made him arrive on time.

The agent possibly also has (in the profile of $f$ ) information about the degree to which $\mathrm{f}$ is likely to make counterfactual reasoning after a failure (not all people are equally likely to do so).

\footnotetext{
${ }^{9}$ To get all useful information, the personal assistant agent may develop several strategies: communicate with the car agent, question the father or the personal assistant of people knowing him, make deductions... but this is not the point here.
} 


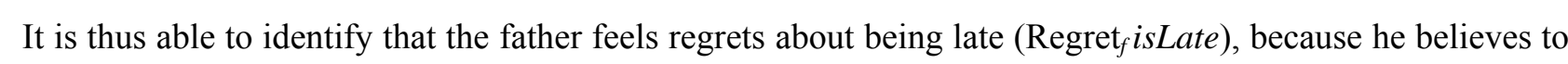

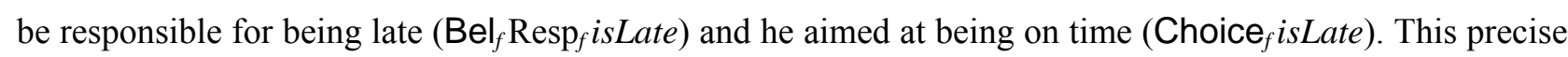
belief about f's emotion allows the agent to behave in the best manner, for example by adopting adapted strategies to help $f$ to cope with his regret. Our aim is not to formalize these strategies, but rather to present them as illustrations showing the importance for the agent to have as much information as possible about the nature and object of the emotion.

The agent can for example try to free $f$ from his responsibility for being late: indeed he was not able to foresee the accident, and without this accident he would have arrived on time. It can thus switch the responsibility from $f$ to the bad driver who provoked the accident, or to people who did not clean the road quickly enough after the accident; by this way $f$ 's emotion switches from regret to anger toward the new supposed accountable people. Moreover the agent can exhibit statistics showing that taking car is usually quicker than taking the subway. Finally it can check if the subway has really arrived on time, since if it is not the case it can inform $f$ that he is not responsible for being late because his other possible decision would have led to the same result.

The agent can also play down the importance of the delay (a delay of only five minutes is quite few) and compare it with the advantages of taking car over taking subway (less stress, calm travel, possibility to listen to music...). The agent cannot imagine what would have gone wrong in subway, but it can refer to an history of past travels in subway that have gone wrong for various reasons: someone spilled coffee on $f$ 's suit, subway was late because of strikes, accidents, suicide on the railway... We can even suppose that $f$ 's personal assistant is able to communicate with other users' personal assistants to establish statistics about all that can go wrong in the subway.

\subsection{Scenario 2: guilt}

Tonight it is the son's birthday dinner. He has requested his favorite meal: a roasted chicken. The intelligent fridge has thus proposed a menu taking into account both the dietetic aspects and the family's tastes. Since 
the father will get back home late tonight due to an important meeting, the mother $m$ was assigned to buy what is needed and to prepare the dinner. After an exhausting day's work, the mother has forgotten to buy the roasted chicken, and she has no more time now to go back to the supermarket. She feels guilty because she was responsible for the shopping and by her fault the dinner will be different from what her son has requested.

We consider that the agent embodied in the intelligent fridge is able to communicate with the cupboards in the whole kitchen in order to know their content. It is also able to prepare the menus for the meals of the week, and to establish the corresponding shopping list. It is thus able to deduce that a roasted chicken is missing to prepare the intended dinner.

Actually, the lack of roasted chicken may have resulted from the mother's decision to change the menu, or from an unavailability of this product at the supermarket, what the agent cannot tell at this point. If the agent tries to predict $m$ 's emotion from the limited information it has, it may thus fail and adopt unadapted behavior. However, if it first detect a negative emotion in $m$, and then uses it as additional information, his behavior will be much more relevant to the situation.

The agent thus detects a negative valence in $m$ informing it of a problem. It then tries to explain it from its knowledge and its model of emotions. In particular this agent knows the following information:

- a roasted chicken is missing (but it does not know why yet), preventing the mother to cook the requested dinner $\left(\mathrm{Bel}_{m} \neg\right.$ dinnerAsRequested $)$;

- $m$ was assigned to buy the chicken, thus it is her fault that the dinner will not be as expected $\left(\mathrm{Bel}_{m} \neg \mathrm{Stit}_{A G T \backslash\{m\}} \neg\right.$ dinnerAsRequested);

- there is a rule in the family specifying that the person who celebrates his birthday chooses the menu of the dinner; thus it is ideal for $m$ and for the other inhabitants that the dinner matches the son's request (Ideal ${ }_{m}$ dinnerAsRequested). 
From this information, the agent deduces that the dinner will not be the planned one (Bel $\neg \neg$ dinnerAsRequested). Moreover, since $m$ is responsible for this situation $\left(\operatorname{Resp}_{m}\right.$ $\neg$ dinnerAsRequested) that violates an ideal of the family (Ideal ${ }_{m}$ dinnerAsRequested), the agent can deduce that $\mathrm{m}$ may feel guilty about this (Guilt ${ }_{m} \neg$ dinnerAsRequested). This emotion explains the negative valence measured by the agent. Having identified precisely the mother's negative emotion, the agent can now adopt adequate strategies to improve the mother's well-being. In particular, it can help her to cope with her guilt by proposing either some material strategies (e.g. compensating for her forgetting) or emotional ones (e.g. looking on the bright side of the situation).

Being aware of everything available in the kitchen, the intelligent agent can propose another ingredient to replace the missing one, for example there is some ham in the fridge. It can also propose another recipe that is possible with what is available at home and that still matches the son's preferences in order to ease his disappointment.

The intelligent fridge could also help the mother to look on the bright side of the situation. For example, she hates roasted chicken and her involuntary omission will lead her to prepare another recipe that the whole family could enjoy. This could also be the occasion to go to the restaurant instead and to discover new meals, or to eat roasted chicken without having to prepare it herself.

Please note again that it is important for the agent to have maximal information about $m$ 's mental attitudes and emotion, since the strategy would not be the same if roasted chicken was unavailable at the supermarket, or if she had decided by herself to change the menu.

\section{Conclusion}

In this chapter, we have shown the importance of emotions for Ambient Intelligence. Moreover, we have argued that existing approaches and tools in AmI are not adapted for reasoning about emotions or identifying the object of a user's emotions. We have shown that these limitations are even more obvious 
when looking at complex emotions based on sophisticated forms of reasoning such as reasoning about responsibility and counterfactual reasoning

We have proposed a logical framework for the analysis and formal specification of emotions. In fact, we think that logic is a natural tool for reasoning about the cognitive constituents of complex emotions and for dealing with the identification of the object of emotions. Our logical framework allows to specify the mental attitudes of agents (beliefs, choices, ideals) and to characterize the concept of responsibility which is a fundamental building block of complex emotions. We have also provided a logical characterization of two specific complex emotions based on responsibility: regret and guilt.

Finally, we have presented and formalized two AmI scenarios involving regret and guilt. We have taken into consideration various aspects of emotions relevant to AmI, such as the identification of a user's emotion or the influence of the user's emotion on reasoning and planning.

The present work is a first step towards a general logical model of complex emotions to be applied to Ambient Intelligence. We would like to highlight that what we have proposed in this chapter is a model, in the sense of "an abstract construction that allows to understand a reference system by answering some questions about it." [60]. Note that the use of logic as a modeling language allows us to provide an unambiguous model of (complex) emotions and their links with mental attitudes; such a model is also independent of the implementation language. The implementation of a conceptual model is a huge and hard task and this step remains out of the scope of this chapter. Logic has to be considered as a useful specification language to represent Ambient Intelligence concepts. Some dedicated agent programming languages could be used to improve this step such as 3APL [19] or CLAIM [25]. This could be an interesting continuation of the work presented in this chapter. Another envisaged continuation will be to extend our analysis to other complex emotions such as shame, reproach, relief or embarrassment. 


\section{Acknowledgement}

Carole Adam would like to acknowledge the support of the Australian Research Council, and RealThing Pty

Ltd. under Linkage Grant LP0882013. Benoit Gaudou would like to acknowledge the support of the French IRD SPIRALES research program and the French 3Worlds ANR project. Dominique Longin and Emiliano Lorini are supported by the French ANR project CECIL about 'Complex Emotions in Communication, Interaction and Language' (WWW . irit . fr/CECIL), contract No. ANR-08-CORD-005, and by University of Toulouse through the project AmIE 'Ambiant Intelligence Entities' (scientific call of the Paul Sabatier University - Toulouse III).

\section{References}

[1] E. Aarts and B. Eggen, editors. Ambient Intelligence in Home-Lab. Neroc, Eindhoven, The Netherlands, 2002.

[2] Carole Adam. Emotions: from psychological theories to logical formalization and implementation in a BDI agent. PhD thesis, INP Toulouse, France, 2007.

[3] Carole Adam, Benoit Gaudou, Andreas Herzig, and Dominique Longin. A logical framework for an emotionally aware intelligent environment. In AITAmI'06. IOS Press, 2006.

[4] Carole Adam and Dominique Longin. Endowing emotional agents with coping strategies: from emotions to emotional behaviour. In IVA'2007, volume 4722 of LNCS, pages 348-349. Springer, 2007.

[5] Carole Adam, Andreas Herzig and Dominique Longin. A logical formalization of the OCC theory of emotions. In: Synthese, Springer, Vol. 168:2, p. 201-248, 2009.

[6] R. Alur and T. Henzinger. Alternating-time temporal logic. Journal of the ACM, 49:672-713, 2002.

[7] J.-C. Augusto, P. McCullagh, V. McClelland, and J.-A. Walkden. Enhanced healthcare provision through assisted decision making in a smart home environment. In AITAmI07, 2007.

[8] N. Belnap, M. Perloff, and M. Xu. Facing the future: agents and choices in our indeterminist world. Oxford University Press, New York, 2001. 
[9] L.F. Berkman, T. Glass, I. Brissette, and T.E Seeman. From social integration to health: Durkheim in the new millennium. Social Science and Medicine, 51:843-857, 2000.

[10] Cynthia Breazeal and B. Scassellati. How to build robots that make friends and influence people. In IROS, 1999.

[11] J. Broersen, A. Herzig, and N. Troquard. Embedding alternating-time temporal logic in strategic STIT logic of agency. Journal of Logic and Computation, 16(5):559-578, 2006.

[12] D. Burton. Do anxious swimmers swim slower? Reexamining the elusive anxiety-performance relationship. Journal of sport and exercise psychology, 10:45-61, 1988.

[13] H. N. Castaneda. Thinking and Doing. D. Reidel, Dordrecht, 1975.

[14] C. Castelfranchi and E. Lorini. Cognitive anatomy and functions of expectations. In IJCAI03 Workshop on Cognitive Modeling of Agents and Multi-Agent Interactions. Morgan Kaufmann Publisher, 2003.

[15] B. F. Chellas. Modal logic: an introduction. Cambridge University Press, Cambridge, 1980.

[16] Philip R. Cohen and Hector J. Levesque. Intention is choice with commitment. Artificial Intelligence Journal, 42(2- 3):213-261, 1990.

[17] R. Conte and C. Castelfranchi. Cognitive and social action. London University College of London Press, London, 1995.

[18] Antonio R. Damasio. Descartes' error: emotion, reason, and the human brain. Putnam pub. group, 1994.

[19] Mehdi Dastani, M. Birna van Riemsdijk, and John-Jules Ch. Meyer. Programming Multi-Agent Systems in 3APL. In: Multi-Agent Programming: Languages, Platforms and Applications, pages $39-67,2005$.

[20] F. de Rosis, C. Pelachaud, I. Poggi, V. Carofiglio, and B. D. Carolis. From Greta's mind to her face: modelling the dynamics of affective states in a conversational embodied agent. International Journal of Human-Computer Studies, 59(1-2):81-118, 2003.

[21] K. Ducatel, M. Bogdanowicz, F. Scapolo, J. Leijten, and J.-C. Burgelman. Scenarios for ambient intelligence in 2010. ISTAG report at IPTS Seville, 2001. 
[22] Paul Ekman. Basic emotions. In T. Dalgleish and M. Power, editors, Handbook of cognition and emotion. John Wiley \& Sons, 1999.

[23] Paul Ekman. An argument for basic emotions. Cognition and Emotion, 6:169-200, 1992.

[24] M. El Jed, N. Pallamin, J. Dugdale, and B. Pavard. Modelling character emotion in an interactive virtual environment. In AISB 2004 Symposium: Motion, Emotion and Cognition, 2004.

[25] Amal El Fallah-Seghrouchni and Alexandru Suna. CLAIM and SyMPA: A Programming Environment for Intelligent and Mobile Agents. In: Multi-Agent Programming: Languages, Platforms and Applications, pages 95-122, 2005.

[26] Clark Elliott, Jeff Rickel, and James Lester. Lifelike pedagogical agents and affective computing: An exploratory synthesis. LNCS, 1600:195-211, 1999.

[27] Fabrice Forest, Astrid Oehme, Karim Yaici, and Celine Verchere-Morice. Psycho-social aspects of context awareness in ambient intelligent mobile systems. In 15th IST Summit 2006, 2006.

[28] N. H. Frijda. The Emotions. Cambridge University Press, 1986.

[29] Jonathan Gratch and Stacy Marsella. A Domain-independent Framework for modelling Emotions. Journal of Cognitive Systems Research, 5(4):269-306, 2004

[30] J. Hintikka. Knowledge and Belief. Cornell University Press, New York, 1962.

[31] J. F. Horty and N. Belnap. The deliberative STIT: A study of action, omission, and obligation. Journal of Philosophical Logic, 24(6):583-644, 1995.

[32] John F. Horty. Agency and Deontic Logic. Oxford University Press, Oxford, 2001.

[33] D. Hume. A Treatise of Human Nature. Clarendon Press, Oxford, 1978. Selby-Bigge, L. A. \& Nidditch, P. H. (Eds.)

[34] Roger Jianxin Jiao, Qianli $\mathrm{Xu}$, and Jun Du. Affective human factors design with ambient intelligence. In HAI, 2007.

[35] P. N. Johnson-Laird and K. Oatley. Basic emotions: a cognitive science approach to function, folk theory and empirical study. Cognition and Emotion, 6:201-223, 1992.

[36] D. Kahneman. Varieties of counterfactual thinking. In N. J. Roese and J. M. Olson, editors, What might have been: the social psychology of counterfactual thinking, pages 375-396. Erlbaum, 
Mahwah, NJ, 1995.

[37] Richard S. Lazarus. Emotion and Adaptation. Oxford University Press, 1991.

[38] E. Lorini and C. Castelfranchi. The cognitive structure of surprise: looking for basic principles. Topoi: an International Review of Philosophy, 26(1):133-149, 2007.

[39] E. Lorini and A. Herzig. A logic of intention and attempt. Synthese, 163(1):45-77, 2008.

[40] E. Lorini and F. Schwarzentruber. A Logic for Reasoning about Counterfactual Emotions. In C. Boutilier (Eds.), Proceedings of the Twenty-first International Joint Conference on Artificial Intelligence (IJCAI’09), AAAI Press, pages 867-872, 2009.

[41] G. Marreiros, R. Santos, C. Ramos, J. Neves, P. Novais, J. Machado, and J. Bulas-Cruz. Ambient intelligence in emotion based ubiquitous decision making. In AITAmI’07, 2007.

[42] Stacy Marsella and Jonathan Gratch. Modeling coping behavior in virtual humans: don’t worry, be happy. In AAMAS-2003, pages 313-320. ACM, 2003.

[43] H. McCann. Settled objectives and rational constraints. American Philosophical Quarterly, 28:25$36,1991$.

[44] Astrid Oehme, Antje Herbon, Stefan Kupschick, and Eric Zentsch. Physiological correlates of emotions. In AISBO07, 2007.

[45] A. Ortony. On making believable emotional agents believable. In R. Trappl, P. Petta, and S. Payr, editors, Emotions in Humans and Artifacts, pages 189-212. MIT Press, 2003.

[46] Andrew Ortony, G.L. Clore, and A. Collins. The cognitive structure of emotions. Cambridge University Press, Cambridge, MA, 1988.

[47] Marc Pauly. A modal logic for coalitional power in games. Journal of Logic and Computation, 12(1):149-166, 2002

[48] Rosalind W. Picard. Affective Computing. MIT Press, 1997

[49] Rosalind W. Picard, E. Vyzas, and J. Healey. Toward machine emotional intelligence: analysis of affective physiological state. IEEE transactions pattern analysis and machine intelligence, 23(10), 2001.

[50] Helmut Prendinger and Mitsuru Ishizuka. Social role awareness in animated agents. In Proceedings 
of AGENTS 2001, pages 270-277, 2001.

[51] Helmut Prendinger and Mitsuru Ishizuka. Human physiology as a basis for designing and evaluating affective communication with life-like characters. IEICE Transactions on Information and Systems, E88-D(11):2453-2460, 2005.

[52] Anand S. Rao and Michael P. Georgeff. Modeling rational agents within a BDI-architecture. In Proceedings of KR'91, pages 473-484. Morgan Kaufmann Publishers, 1991.

[53] J. Rickel and W. Lewis Johnson. Steve: an animated pedagogical agent for procedural training in virtual environments. Animated Interface Agents: making them intelligent, pages 71-76, 1997.

[54] K. R. Scherer. Appraisal Processes in Emotion : Theory, Methods, Research, chapter Appraisal Considered as a Process of Multilevel Sequential Checking, pages 92-120. Oxford University Press, New York, 2001.

[55] John R. Searle. Intentionality: An essay in the philosophy of mind. Cambridge University Press, 1983.

[56] N. Sebe, I. Cohen, T. Gevers, and Huang T. S. Multimodal approaches for emotion recognition: a survey. In SPIE: Internet Imaging, pages 56-67, 2004.

[57] Bas Steunebrink, Mehdi Dastani, and John-Jules Meyer. A logic of emotions for intelligent agents. In R.C. Holte and A.E. Howe, editors, Proc. AAAI-07, pages 142-147, 2007. AAAI Press.

[58] J. M. Susskinda, G. Littlewortb, M. S. Bartlettb, J. Movellanb, and A. K. Anderson. Human and computer recognition of facial expressions of emotion. Neuropsychologia, 45:152-162, 2007.

[59] J. P. Tangney. The self-conscious emotions: shame, guilt, embarrassment and pride. In T. Dalgleish and M. Power, editors, Handbook of cognition and emotion. John Wiley \& Sons, 1999.

[60] J-P. Treuil, A. Drogoul and J-D. Zucker. Modélisation et simulation à base d'agents : Approches particulaires, modèles à base d'agents, de la mise en pratique aux questions théoriques. Dunod, 2008.

[61] Jan Treur. On human aspects in ambient intelligence. In Proceedings of First International Workshop on Human Aspects in Ambient Intelligence (HAI), pages 5-10, 2007.

[62] A. van Breemen. iCat: Experimenting with animabotics. In AISB, pages 27-32, University of 
Hertfordshire, Hatfield, UK, April 2005.

[63] Kazuyoshi Wada and Takanori Shibata. Robot therapy in a care house -its sociopsychological and physiological effects on the residents. In ICRA06, 2006.

[64] Chen Wu and Hamid Aghajan. Context-aware gesture analysis for speaker hci. In AITAmI'08, 2008.

[65] M. Zeelenberg and E. van Dijk. On the psychology of "if only": regret and the comparison between factual and counterfactual outcomes. Organizational Behavior and Human Decision Processes, 97(2):152-160, 2005.

[66] Jiehan Zhou, J. Riekki, Y. Changrong, and E. Kärkkäinen. AmE framework: a model for emotionaware ambient intelligence. In ACII2007: Doctoral Consortium, 2007.

\section{Appendix: Definitions}

Agent. Autonomous entity that is able to perceive its environment, reason about it, maintain personal goals, plan appropriate ways to achieve them, and act accordingly to modify in turn its environment. Some agents are also able to communicate with other artificial agents or with humans, or to express and understand emotions.

Emotion. Intentional affective mental state (in the sense of Searle, i.e. having an object) that arises automatically as a result of an individual's interpretation of his environment and its relation to his attitudes (e.g. goals and interests). Emotions can then influence behavior in various ways, and were thus long considered irrational before research showed that they were actually essential in rational decision making.

Mood. Non-intentional affective mental state (in the sense of Searle, i.e. having no object). For example, one can be in a happy mood (about nothing in particular) which is different from feeling the emotion of happiness (about something, for example that the weather is sunny). Moods are also characterized by a 
longer duration than emotions.

Cognitive appraisal theories of emotions. In these theories, emotions are triggered by a process (called appraisal) determining the significance of the situation for the individual by assessing it w.r.t. various criterions called appraisal variables (e.g. desirability).

Appraisal variables. Criteria used to evaluate the significance of a situation w.r.t. an individual's goals, ideals or desires, determining the triggered emotion. For example a pleasant situation (the pleasantness variable is measured by comparing the situation with the individual's goals) leads to a positive emotion (e.g joy).

Complex emotions. Emotions based on sophisticated forms of reasoning such as reasoning about responsibility and counterfactual reasoning. They are thus based on a larger set of appraisal variables than the set involved in basic emotions.

Counterfactual reasoning. Reasoning based on what the current state of the world would be or would not be if I had acted differently, i.e. if I had performed or had not performed a certain action. For example, if I had slept this morning, I would be less tired; if I had not run this morning, I would feel sad; if I had taken the train instead of my car, I would not be caught in a traffic jam; if I had bought petrol earlier, I would not have run out of petrol on the freeway.

Coping strategies. Strategies (or actions sets) used by an agent to restore its well-being in stressful situations. For example one can deny a piece of information that makes him too sad; one often starts by denying the death of a loved one.

Actions tendencies. Processes associated to emotions that allow individuals to make quick decisions, using their emotions as a kind of heuristic. For example, fear induces a tendency to escape while anger induces a 
tendency to attack.

BDI logic. Formal language used to represent agents' reasoning in terms of their mental attitudes (beliefs, desires, intentions) as described by philosophers of mind (e.g. Bratman).

Responsibility. An agent $i$ is responsible for causing $p$ if and only if $i$ has performed a certain action and if $i$ had not performed this action then $p$ would not be true now. For example a driver is responsible for causing an accident by running a red light because if he had not, then the accident would not have occurred. 